\title{
Caracterización de un modelo de sistema de información interorganizacional para el sector de la edificación domótica
}

\author{
Antonio Pereira-Rama ${ }^{1}$, Julián Chaparro-Peláez ${ }^{1}$, Ángel Francisco Agudo-Peregrina ${ }^{1}$ \\ antonio.pereira.rama@alumnos.upm.es,julian.chaparro@upm.es, af.agudo@upm.es \\ ${ }^{1}$ Departamento de Ingeniería de Organización, Administración de Empresas y Estadística, Universidad \\ Politécnica de Madrid, 28040, Madrid, España.
}

DOI: 10.4304/risti.10.81-96

\begin{abstract}
Resumen: El sector de la edificación es uno de los principales sectores económicos con grandes repercusiones en el conjunto de la sociedad y en los valores culturales que entraña el patrimonio arquitectónico y, sin embargo, carece de una regulación acorde. La imparable evolución de las TIC y la cultura de la competencia han provocado la aparición de nuevos servicios y operadores. Por ello, el presente estudio, basado en un trabajo de investigación empírico, propone la caracterización de un sistema de información interorganizacional (SIIO) entre los diversos agentes que configuran la cadena de valor del sector de la edificación. El objetivo es la mejora de gestión en las interrelaciones entre los diferentes agentes de cara a abordar proyectos de edificación más complejos, contribuyendo, en particular, a la mejora de la calidad de la vivienda y, por tanto, a la calidad de vida de la sociedad en general.
\end{abstract}

Palabras-clave: Sector de la Edificación; SIIO; TIC; PYME; Domótica.

\begin{abstract}
The design, build and management of housing is one the largest industries in the world. The complexity of the process for managing all of information related to this industry has resulted in tremendous inefficiencies, leading to massive money and time losses. The information and knowledge gathered in one phase of the process is frequently stored in multiple locations, involves thousands of people and is rarely transferred. The rapid evolution of ebusiness, introduced in the past few years, offers new ways for organizations to perform tendering processes and participate in bidding. This study, based on an empirical research work, proposes an interorganizational information system (IOIS) in order to improve knowledge and value transference in the sector. The implementation of an IOIS in the construction sector as a best-practice is the last goal of this investigation.
\end{abstract}

Key-words: Construction; IOIS; ICT; SME; Domotic services. 


\section{Introducción}

Los problemas derivados la falta de regulación existente en el sector de la edificación son motivados generalmente por otro problema: la falta de información de los agentes que intervienen en el mismo. Esto se traduce en que proyectos de finita duración se alargan inexplicablemente; y, en otros casos, cuando las prisas apremian, se suelen realizar en menos tiempo del requerido y, por tanto, sin cumplir con los requisitos de calidad que deberían tener.

Conseguir la máxima eficiencia en el sector de la edificación, pasa, como en todos los sectores productivos, por la necesidad de optimizar todos los procesos internos que consumen recursos. Es necesario conseguir que los objetivos se cumplan, pero, a la vez, hay que poner el mayor énfasis en la tarea para que en su consecución se consuma la menor cantidad de recursos posible, es decir, hay que minimizar los costes sin que por ello disminuyan los objetivos.

Existe una clara tendencia en el sector de la edificación a mejorar la gestión de la información como herramienta para optimizar los recursos. En este sentido podemos encontrar propuestas de sistemas de comercio electrónico orientados a la colaboración e intercambio de información asociada al proyecto (Yeung et al., 2009); propuestas de sistemas de gestión de la cadena de suministro mediante Marketplaces B2B (CastroLacouture et al., 2007; Chung et al., 2009; Carbonell-Ureña, 2012); y propuestas de sistemas para la gestión de información en el hogar y el soporte a su mantenimiento, como la tecnología BIM (Building Information Modelling) el formato IFC (Industry Foundation Classes) y su asociación a los sistemas CAD (Vanlande et al., 2008; Shin et al., 2008; Jeong et al., 2009).

Es por ello que un sistema de información interorganizacional (SIIO) que controle el flujo de información entre sus diferentes agentes integrantes no sólo permitirá sufragar estas lagunas, sino que además fomentará la colaboración y especialización de agentes y un seguimiento del proyecto más interactivo.

\section{Marco teórico}

La complejidad del entorno actual obliga a las empresas del sector de la edificación a adaptarse rápidamente para sobrevivir en el mercado. En especial, para las PYMEs, que muchas veces carecen de los recursos necesarios para enfrentarse a estos retos, surgen nuevas necesidades de cooperación e intercambio de información entre organizaciones.

\section{Los retos actuales del sector de la edificación}

El sector de la edificación ha experimentado una caída considerable en los últimos años. La retracción del mercado ha producido que se ralentice el ritmo de la edificación, ritmo que hasta el momento era frenético y que empleaba a miles de trabajadores. Sin embargo, esta retracción ha estado contenida, en parte, motivada por la existencia de muchas PYMEs que, gracias a su flexibilidad, han sabido y podido adecuarse mejor que las grandes empresas a los reajustes del mercado inmobiliario. En concreto, muchas de estas PYMEs se han especializado en la construcción de viviendas unifamiliares, restauraciones o reformas integrales (BIC-GALICIA, 2010), existiendo 
una importante apuesta por aplicar la domótica a las viviendas y mejora de las instalaciones, así como la utilización de energías renovables y uso de materiales geotérmicos, que se configuran como aspectos claves para recuperación del sector (Cinza-Cabarcos, 2008).

Una vivienda domótica es aquella que permite una mayor calidad de vida a través de la tecnología, ofreciendo una reducción del trabajo doméstico, un aumento del bienestar y de la seguridad de sus habitantes, y una racionalización de los distintos consumos. En la actualidad, las familias necesitan vivir en entornos más flexibles para compaginar, de la mejor forma posible, sus tareas domésticas con otras áreas de su vida, como el campo profesional o el del ocio, lo cual permite concebir el hogar como un hogar evolucionado para la prestación de servicios de valor añadido, $\mathrm{y}$, en definitiva, surge el concepto de hogar digital (Telefónica, 2004). Por tanto, el desarrollo del mercado de la domótica no es un problema tecnológico sino básicamente una cuestión de servicios y modelos de negocio que permitan ofrecer estos servicios a un precio asequible (CastroGonzález, 2008).

La actividad de la edificación posee características especiales y únicas entre todas los distintos tipos de industrias. Cada edificación es única y diferente, en el sentido de que representa un esfuerzo especialmente dirigido a desarrollar un proyecto concreto. En este esfuerzo, es necesario primero concebir claramente el objetivo para diseñar el proyecto de una manera detallada y práctica, posteriormente se construyen los elementos diseñados y finalmente se ponen en funcionamiento. Cuanto más servicios avanzados se incorporen, como los que ofrece la domótica, más complejo será este proyecto y contará con la participación de un mayor número de agentes, con la consiguiente dificultad en su correcta coordinación para conseguir los objetivos deseados optimizando los recursos disponibles (Chung et al., Kumaraswamy \& Palaneeswaran, 2009).

\section{La cooperación organizativa y el SIIO}

Las relaciones interorganizativas constituyen un fenómeno que está adquiriendo mayor importancia y en el que cada vez se están involucrando un mayor número de organizaciones de distinta naturaleza. Las razones de la cooperación se encuentran, principalmente, en la turbulencia del entorno competitivo, caracterizado por un alto grado de dinamismo tecnológico y por la internacionalización y globalización de la economía. Por tanto, la cooperación constituye una de las fórmulas más dinámicas para conseguir la masa crítica necesaria para que la PYME, a pesar de su pequeño tamaño relativo, pueda resultar competitiva e, integrada en las redes tecnológicas, pueda acceder a los mercados exteriores sin elevado riesgo y con un coste soportable (Lockett \& Brown, 2006).

Los sistemas de información alcanzan actualmente un alto grado de penetración en los procesos internos de las empresas, facilitando los intercambios de información incluso con otras empresas. En este entorno surgen los sistemas de información interorganizacionales (SIIO) (Orero-Giménez \& Criado-Fernández, 1999).

Los SIIOs representan la transición de la competencia entre empresas a la cooperación entre empresas. A medida que las organizaciones tienden a relaciones económicas de cooperación, las TIC y los SIIOs desempeñan un papel que permite que la transición 
sea posible. Por tanto, el SIIO puede verse como la interfase para la interacción entre las TIC y la relación interorganizacional. Sin embargo, los argumentos racionales y económicos, junto con la factibilidad técnica, no son suficientes para consumar la alianza de colaboración, siendo necesario considerar aspectos sociopolíticos, tales como la "química personal" e interacción entre directivos de las organizaciones, y la compatibilidad entre las propias organizaciones en aspectos culturales, filosóficos y estratégicos, que determinan, en su conjunto, si la alianza se hará efectiva y madurará. El modelo de caracterización propuesto a continuación refleja la relación cooperativa entre organizaciones (Orero-Giménez \& Criado-Fernández, 1999).

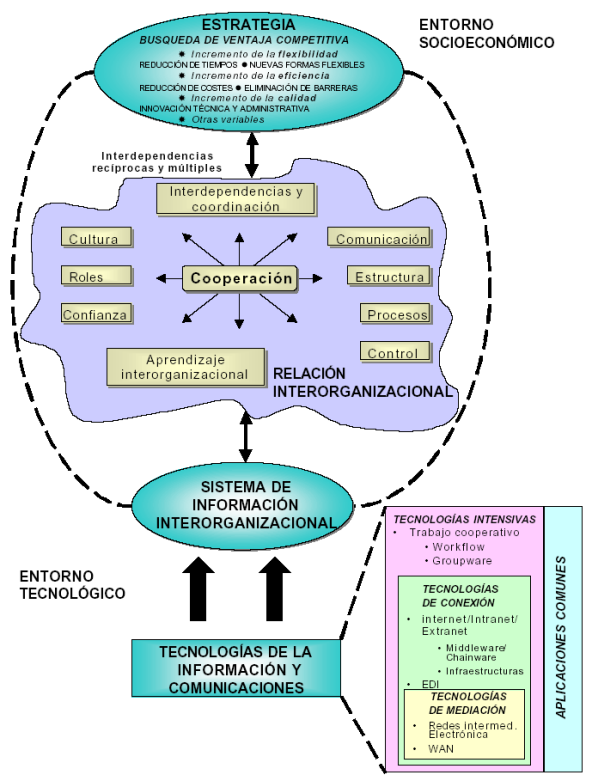

Fuente: Orero Giménez y Criado Fernández, 1999

Figura 1 - Modelo de caracterización de un SIIO

De esta interfase entre la relación interorganizacional y las TIC se derivan las dimensiones de un SIIO que resumimos brevemente a continuación:

- Dimensión estratégica: caracteriza el potencial de los SIIOs como fuente de ventajas competitivas.

- Dimensión colaborativa: potencia los canales de comunicación entre empresas y los SI que alimentan el proceso de toma de decisiones.

- Dimensión organizativa: desde la perspectiva de organización global e implica caracterizar las interdependencias entre participantes.

- Dimensión tecnológica: caracteriza las funcionalidades de las tecnologías requeridas.

Criado-Fernández (2000) considera estas dimensiones para la construcción del modelo de análisis de virtualidad y potencial de virtualización (Criado-Fernández, 200o) con el que se afirma la capacidad del SIIO como posibilitador de la organización virtual y el 
traslado de las ventajas competitivas proporcionadas y sustentadas por los SIIO a la organización virtual.

\section{Metodología}

El presente estudio, basado en un trabajo de investigación, pretende proponer la caracterización de un modelo de SIIO para el sector de la edificación domótica.

\section{Propuesta de un SIIO para el sector de la edificación domótica}

En los últimos años, han aparecido muchos trabajos relacionados con la aplicación de los sistemas de comercio electrónico B2B (White, Daniel, Ward \& Wilson, 2007). En general todos los trabajos se centran en considerar una organización como orquestadora de la relación interorganizacional y la capacidad para introducir mejoras en la cadena de suministro para el aprovisionamiento de materiales para la edificación, mostrando las ventajas del modelo electrónico frente al tradicional (Castro-Lacouture, Medaglia \& Skibniewski, 2007).

Derivado de las necesidades expuestas en el marco teórico y atendiendo al modelo de caracterización de un SIIO (Orero-Giménez \& Criado-Fernández, 1999) proponemos, a continuación, un SIIO para el sector de la edificación domótica.

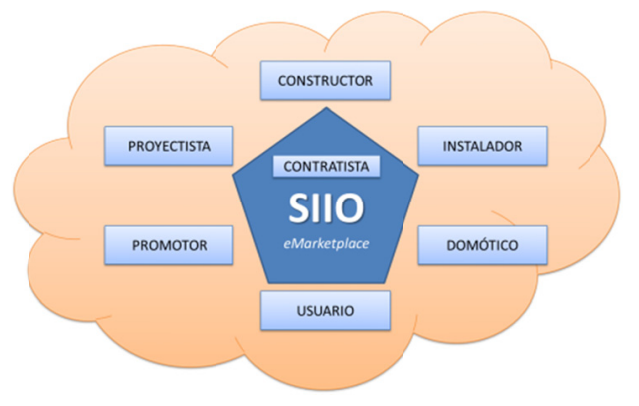

Figura 2 - SIIO para el sector de la edificación domótica

El SIIO para el sector de la edificación domótica sería un sistema de información que permita a los diferentes agentes del sector la compartición de datos (relativos a proyectos y recursos) y software; y todo ello fuera de las fronteras de la organización. El SIIO contaría con las siguientes características en cuanto a su configuración:

- Una empresa externa que se encargaría de la gestión, alojamiento y dimensionamiento del SIIO. Esta empresa externa bien podría ser una empresa participada por todos los agentes que componen el SIIO.

- Proporcionaría aplicaciones para complementar los sistemas de información de los agentes participantes.

- El usuario de la vivienda es también un participante del SIIO.

- El rol del contratista quedaría asumido por el SIIO, siendo necesario un replanteamiento del modelo de negocio de este agente. 
Atendiendo a la dimensión estratégica se propone la gestión virtual de los procesos de oferta y demanda en el sector de la edificación considerando tres roles principales (Kerridge, Halaris \& Mentzas, 2000): cliente, contratista y proveedor según se recoge en la Figura 3.

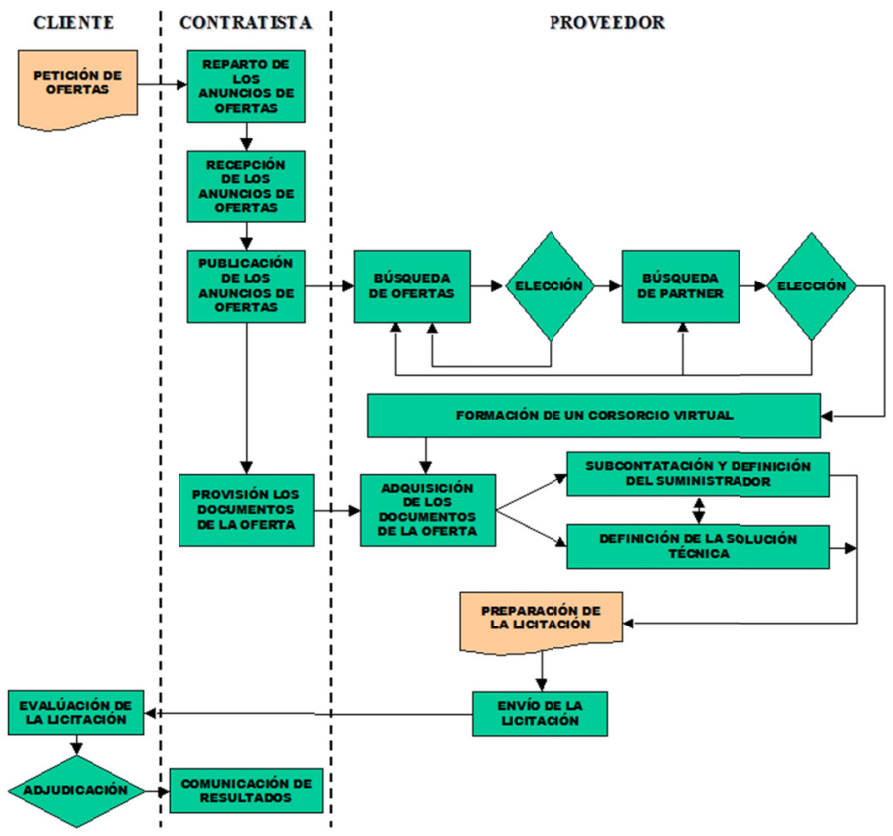

Fuente: Adaptado de Kerridge et al., 2000

Figura 3 - Procesos de oferta y demanda en el sector de la edificación

La gestión virtual de los procesos de oferta y demanda permite pensar en la idea de que el propio SIIO facilite mecanismos electrónicos para soporte de la cadena de suministro (Carbonell-Ureña, 2012). Por tanto, la introducción de un SIIO en el sector de la edificación domótica beneficiaría a todos los miembros de la cadena de suministro, como reflejamos de forma resumida en la siguiente tabla:

Tabla 1 - Beneficios potenciales en la cadena de suministro por la implantación de un SIIO

\begin{tabular}{ll}
\hline Agente & Beneficios potenciales \\
\hline Promotores & Mejora de la eficiencia del proyecto \\
& Reducción de los costes y posibilidades de error \\
& Compresión del proceso de desarrollo \\
\hline Proyectistas & Mejora de la comunicación y Ahorro de Tiempos \\
& Incremento de la precisión y rapidez de especificación \\
\hline Contratistas & Bajos costes de administración y comunicaciones \\
& Ofrecimiento y eficacia de consecución \\
& Ahorro de tiempos \\
\hline
\end{tabular}




\begin{tabular}{ll}
\hline Agente & Beneficios potenciales \\
\hline Más control y seguridad de proyecto \\
& Reafirma la comunicación en el proyecto \\
\hline Constructores & $\begin{array}{l}\text { Bajo inventario y estado real de sus costes } \\
\text { Bajo coste de servicio a clientes }\end{array}$ \\
\hline Domóticos & Reducen los costes de canal \\
& $\begin{array}{l}\text { Mejora el acceso a la información } \\
\text { Rentabilidad de acceso para la compra activa y } \\
\text { especificaciones de los clientes }\end{array}$ \\
\hline
\end{tabular}

Según la dimensión colaborativa, la utilización de las TIC en la cooperación conseguiría reducir las distancias y aumentar las interdependencias entre espacios alejados geográficamente, permitiendo extender la cadena de valor de negocio a áreas geográficas mayores (Pizarro-Moreno, Real \& De la Rosa, 2011).

La dimensión organizativa del SIIO propuesto se basaría en un modelo de consorcio virtual que se define como una cooperación de diferentes organizaciones con capacidad de complementarse las cuales se unen usando las TIC con el objeto de realizar una propuesta, e incluso, conseguir la oportunidad de implementar un proyecto concreto (Kerridge, Halaris, \& Mentzas, 2000).

Por último, la dimensión tecnológica es importante comentar que no existe en el mercado una solución tecnológica completa que satisfaga el modelo propuesto, por ello será necesario asumir un desarrollo a medida para la implementación del SIIO (Schlueter \& Thesseling, 2009) que se articulará en base a los siguientes premisas:

- La infraestructura tecnológica que se plantea es un sistema de eMarketplace, por tanto, sobre la red Internet.

- Existiría un sistema de flujo de trabajo (BPM) unido a un sistema de gestión documental, ya que resulta evidente que la actividad de las organizaciones gira en torno a la ejecución de proyectos.

- El intercambio de información se basará en XML que se está convirtiendo en la norma para almacenar datos que se intercambian entre aplicaciones ya que permite describir cualquier tipo de datos, incluyendo información material, documentos de proyectos, dibujos $\mathrm{CAD}$, información comercial, los documentos, etc. (Kong et al., 2004).

\section{Diseño del modelo de investigación}

Para la validación del SIIO propuesto consideraremos, como punto de partida, el modelo de análisis de virtualidad de Criado-Fernández (2000) a partir del cual diseñaremos el modelo de investigación para caracterizar el SIIO propuesto para el sector de la edificación domótica.

El modelo de análisis de virtualidad recoge la relación entre factores causa y factores de éxito que ofrece el SIIO en el mercado en el que opera la organización (factores efecto). Los factores causa se corresponden con 34 variables independientes de investigación agrupadas según las 4 dimensiones indicadas, estas variables se miden con 146 indicadores que se corresponden con 146 preguntas de investigación. Los factores 
efecto se corresponden con 5 variables dependientes de investigación que se miden con 51 indicadores que se corresponden con 51 preguntas de investigación (CriadoFernández, 2000).

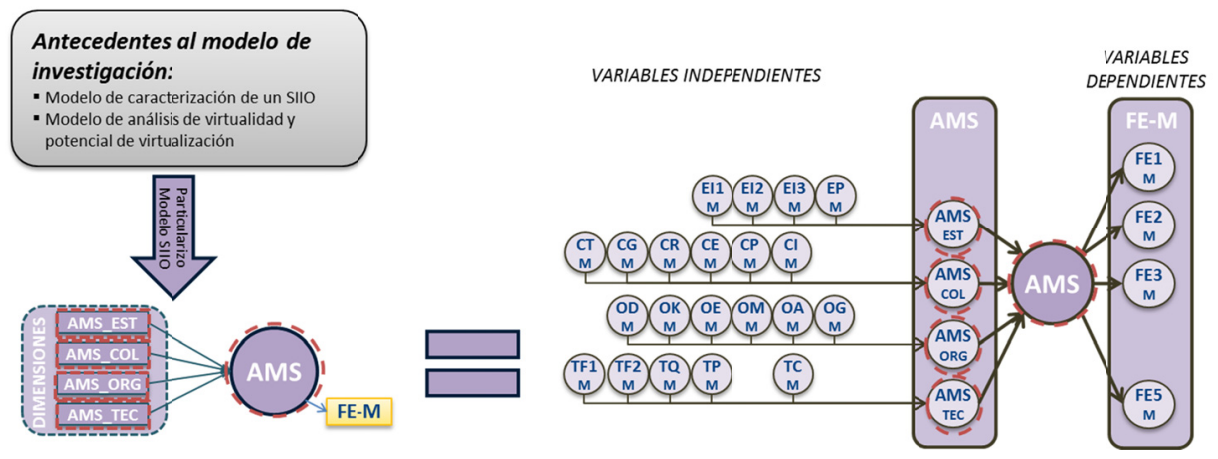

Figura 4 - Modelo inicial de investigación

En este modelo, podemos agrupar los indicadores en tres conjuntos según su aplicación: la empresa, el sector y el SIIO. Si nos quedamos solamente con los indicadores relacionados con el SIIO, obtenemos un modelo que nos permite analizar la adecuación del SIIO (AMS) como fuente y soporte de ventajas competitivas, y que estaría constituido por 21 variables independientes (agrupadas según las 4 dimensiones indicadas y que se miden mediante 75 indicadores) y 4 variables dependientes (que se miden mediante 13 indicadores). Así, obtenemos un modelo inicial de investigación, que recogemos en la Figura 4.

Las variables de este modelo se corresponden, debido a la particularización indicada, con variables procedentes del modelo de análisis de virtualidad y potencial de virtualización, conservando su denominación original (Criado-Fernández, 2000). No obstante, este modelo inicial de investigación presenta un número elevado de variables e indicadores que debe de ser depurado mediante la validación del mismo al objeto concreto de esta investigación y como paso previo antes de utilizarlo para la caracterización del SIIO propuesto.

\section{Validación del modelo de investigación}

Para la validación del modelo de investigación se ha considerado una población compuesta por las empresas del sector de la edificación con sede social en la provincia de La Coruña identificadas según CNAE en la guía ARDAN de empresas de Galicia. En concreto, para la validación, la población considerada era de 894 empresas de las cuales se determinó una muestra aleatoria (nivel de confianza 95\%, error máximo 10\% y $\mathrm{p}=\mathrm{q}=0,5)$ de 88 empresas sobre las que se aplicó un cuestionario, mediante entrevista personal, de 88 preguntas de investigación (correspondientes a los indicadores del modelo). Las entrevistas para la validación de este modelo fueron realizadas entre los meses de febrero y mayo del año 2003. 


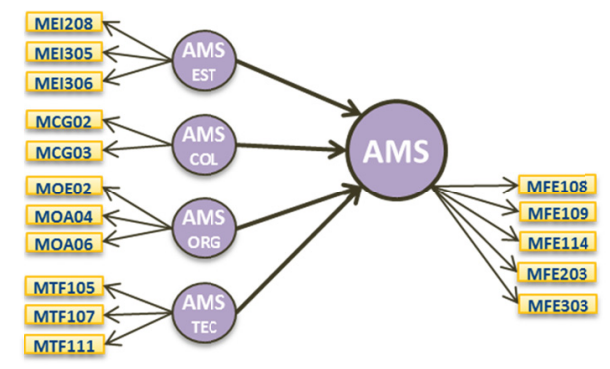

Figura 5 - Modelo de investigación validado

La validación del modelo inicial de investigación se realizó utilizando la técnica PLSPM (Cepeda-Carrión \& Roldán-Salgueiro, 2005), considerando, en primer lugar, el tratamiento de los constructos de segundo orden identificados (Lozano-Santiago, 2007) (identificados con un círculo rojo en la Figura 4) y posteriormente la evaluación de los diferentes modelos de medida resultantes. La aplicación de esta técnica condujo a la depuración de los indicadores, obteniendo un modelo con 4 variables independientes, 1 variable dependiente y 16 indicadores (Figura 5).

Este proceso de validación y depuración de indicadores nos permite definir las variables del modelo de investigación tomando como referencia las variables de partida correspondientes al modelo de análisis de virtualidad y potencial de virtualización de Criado-Fernández (2000):

- AMS (Adecuación del modelo de SIIO, variable dependiente): resultado de identificar la estrategia de la organización, en respuesta a los factores de éxito del mercado en que opera. En concreto, se refiere a flexibilidad y eficiencia organizativa, y reducción de barreras geográficas.

- AMS_EST (Dimensión estratégica, variable independiente): vías o mecanismos que utiliza la organización para disponer factores de producción (recursos materiales, humanos e intangibles), y el enfoque dado a la forma de trabajar o configurar el trabajo.

- AMS_COL (Dimensión colaborativa, variable independiente): atiende al hecho de que la relación esté dispersa geográficamente en ámbito global permite el acceso a mercados y entornos de otra forma inaccesibles.

- AMS_ORG (Dimensión organizativa, variable independiente): se refiere a los aspectos de capacidad de trabajo en equipo y capacidad de aprendizaje interorganizacional o transferencia de conocimiento.

- AMS_TEC (Dimensión tecnológica, variable independiente): es el nivel de calidad de la información disponible y recoge aspectos relacionados con accesibilidad a la información, fiabilidad y facilidad de obtención, grado de uso del soporte electrónico, facilidad de manejo y rapidez de acceso.

De acuerdo con la representación gráfica del modelo (Figura 5) se recogen las relaciones entre estas variables que validarán mediante estudio empírico. 


\section{Dimensionamiento del trabajo de campo}

Para la validación de las relaciones y caracterización del modelo de investigación, el trabajo de campo longitudinal se ha prolongado a lo largo de cinco años adicionales al de la validación. La población considerada está compuesta por las empresas del sector de la edificación con sede social en la provincia de La Coruña determinadas utilizando la guía de empresas ARDAN e identificadas según CNAE, sobre esta población se determinó, para cada uno de los años, una muestra (nivel de confianza 95\%, error máximo $10 \%$ y $\mathrm{p}=\mathrm{q}=0,5$ ) de empresas sobre las que se aplicó un cuestionario, mediante entrevista personal, de 16 preguntas de investigación (correspondientes a los indicadores del modelo validado). En la Tabla 2 recogemos la ficha técnica del dimensionamiento del trabajo de campo realizado.

Tabla 2 - Dimensionamiento del trabajo de campo realizado

\begin{tabular}{lcccccc}
\hline Año & $\mathbf{2 0 0 3}$ & $\mathbf{2 0 0 5}$ & $\mathbf{2 0 0 6}$ & $\mathbf{2 0 0 7}$ & $\mathbf{2 0 0 8}$ & $\mathbf{2 0 0 9}$ \\
\hline Entrevistas & feb - may & mar - ago & ago - sep & ago - oct & sep - oct & mar - abr \\
\hline Muestra & 88 & 116 & 124 & 133 & 140 & 142 \\
\hline
\end{tabular}

El trabajo de campo longitudinal permite valorar la sostenibilidad del modelo en el horizonte temporal de la investigación, extendiendo así la validez del modelo.

\section{Análisis y discusión de los resultados obtenidos}

El análisis y discusión de los resultados se realizará atendiendo tanto al análisis basado en la técnica PLS-PM que permite evaluar las relaciones del modelo, como al análisis basado en estadística descriptiva para la caracterización del modelo.

\section{Validación de las relaciones del modelo}

En la Tabla 3 se recogen los resultados de la evaluación del modelo estructural mediante la aplicación de la técnica PLS-PM a lo largo del horizonte temporal del trabajo de investigación. La evaluación del modelo estructural nos permite obtener la cantidad de varianza explicada por las variables dependientes e independientes, así como los coeficientes y significancia estadística de las relaciones.

Tabla 3 - Evaluación del modelo estructural y relevancia predictiva

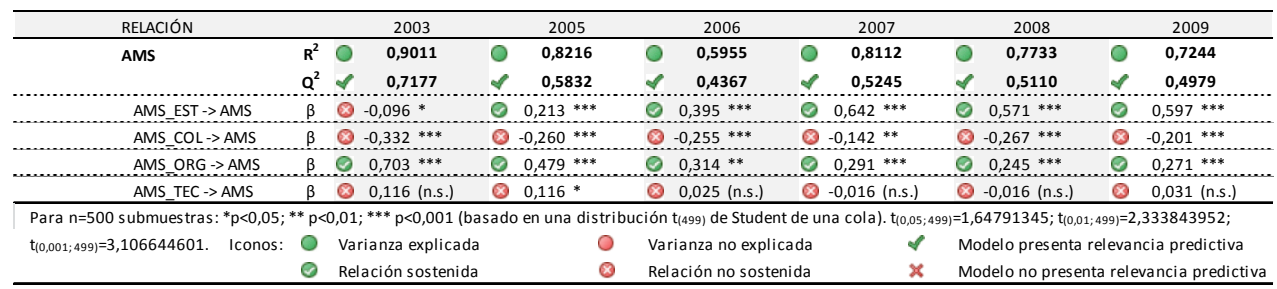

Como se puede observar la varianza explicada es adecuada para AMS (puesto que cumple siempre que $\left.\mathrm{R}^{2} \geq 0,1\right)$, del mismo modo el modelo cuenta con relevancia predictiva $\left(Q^{2}>0\right)$ para todo el horizonte temporal de la investigación. 
En cuanto a las relaciones, observamos que dos relaciones se sostienen mientras que otras dos no se sostienen a lo largo de horizonte temporal. En concreto, podemos afirmar, dada la significancia estadística $\left({ }^{* * *}\right.$ en casi todos los casos de relaciones sostenidas), que el modelo verifica las relaciones AMS_EST $\rightarrow$ AMS y AMS_ORG $\rightarrow$ AMS, y no verifica las relaciones AMS_COL $\rightarrow$ AMS y AMS_TEC $\rightarrow$ AMS. Lo que se nos permite concluir que:

- Los recursos productivos (recursos materiales, humanos e intangibles) que proporciona el SIIO propuesto para el sector de la edificación domótica, así como la forma de trabajar, son considerados por las empresas del sector como una importante fuente de ventajas de flexibilidad y eficiencia organizativa, y permiten la reducción de barreras geográficas.

- Las empresas del sector no consideran que con el SIIO propuesto puedan colaborar si existe dispersión geográfica. Es decir, las empresas ven que la proximidad geográfica es un aspecto necesario para la eficiencia organizativa y el SIIO propuesto no permite superar este aspecto.

- El SIIO propuesto permite potenciar la capacidad de trabajo en equipo y la trasferencia de conocimiento lo que dota a las empresas participantes de ventajas de flexibilidad y eficiencia organizativa.

- Las empresas del sector no consideran la información como un activo estratégico para mejorar la eficiencia organizativa. Por tanto, no perciben como necesaria mejorar la calidad de la información disponible. Lo cual está en consonancia con que las empresas de este sector son muy reacias a la utilización de las TIC.

De estas conclusiones, de cara a mejorar el SIIO propuesto, consideramos dos aspectos de mejora para el SIIO propuesto:

- Las ubicación geográfica de las empresas es una variable que el SIIO debe de considerar a la hora de establecer relaciones de cooperación. Por tanto, se debe de priorizar la relación entre empresas próximas a la ubicación del proyecto frente a otras no tan próximas. En definitiva, la proximidad a la ubicación del proyecto es considerada como un aspecto necesario en la colaboración.

- El SIIO debe de estar más orientado a la organización y contacto comercial que a la gestión documental en sí. Por tanto, previsiblemente, el manejo de documentación, en una primera fase de implantación del SIIO no será un aspecto tan relevante como la gestión de relaciones comerciales.

\section{Caracterización del modelo}

La escala de medición utilizada para los 16 indicadores es un escala de tipo Likert-5 (expresada numéricamente como 1-2-3-4-5 según el grado de rechazo o aceptación) de la cual se ha hecho un escalado al intervalo o-10.

Atendiendo a este escalado en la Tabla 4 se recoge la valoración media para cada uno de los indicadores del modelo. Como se puede observar los valores medios de todos los indicadores que caracterizan el modelo evolucionan a lo largo del tiempo. A pesar de ello, podemos identificar claramente dos tipos de indicadores: los que sistemáticamente mantienen un valor superior a 5, es decir, tiene grado de aceptación y 
caracteriza el modelo); y los de valor inferior a $5 \mathrm{y}$, por tanto, tienen grado de rechazo y no caracterizan el modelo de SIIO propuesto. En estos dos grupos hay indicadores relacionados con las variables independientes y con la variable dependiente.

Tabla 4 - Valoración media de los indicadores para el modelo (2003-2009)

\begin{tabular}{|c|c|c|c|c|c|c|c|c|}
\hline Variable & $\begin{array}{c}\text { ID } \\
\text { Indicador }\end{array}$ & Indicador & 2003 & 2005 & 2006 & 2007 & 2008 & 2009 \\
\hline \multicolumn{9}{|c|}{ Adecuación del modelo de SIIO (AMS) } \\
\hline \multirow{6}{*}{ FE_M } & MFE108 & MEJORAS PLAZO DE ENTREGA & 2,61 & 3,45 & 2,64 & 3,10 & 3,48 & 4,10 \\
\hline & MFE109 & MEJORAS RAPIDEZ NUEVOS PRODUCTOS & 3,84 & 4,20 & 3,79 & 4,00 & 4,55 & 5,12 \\
\hline & MFE114 & MEJORA FACILIDAD ORGANIZACIÓN & 3,18 & 3,64 & 3,23 & 3,50 & 3,93 & 4,86 \\
\hline & MFE203 & MEJORA IMPORTANCIA COSTE & 3,24 & 3,77 & 2,92 & 3,08 & 3,45 & 3,98 \\
\hline & MFE303 & MEJORA DESCENTRALIZACIÓN & 5,60 & 6,70 & 6,39 & 6,67 & 6,30 & 7,02 \\
\hline & MEI208 & USO TIC EN EVALUACIÓN TIC NUEVAS & 5,85 & 6,49 & 5,95 & 6,11 & 6,55 & 7,17 \\
\hline \multirow[t]{2}{*}{ AMS_EST } & MEI305 & USO TIC EN DECISIÓN DESCENTRALIZADA & 3,64 & 4,63 & 4,07 & 4,45 & 4,48 & 5,58 \\
\hline & MEI306 & USO TIC EN APRENDIZAJE COLECTIVO & 4,26 & 4,29 & 3,73 & 4,14 & 4,95 & 5,39 \\
\hline \multirow{2}{*}{ AMS_COL } & MCG02 & RELACIÓN DE ÁMBITO NACIONAL & 5,85 & 7,50 & 8,13 & 8,65 & 8,52 & 8,64 \\
\hline & MCG03 & RELACIÓN DE ÁMBITO GLOBAL & 3,66 & 5,39 & 6,29 & 6,92 & 6,54 & 6,71 \\
\hline \multirow{3}{*}{ AMS_ORG } & MOE02 & PROMOCIÓN DEL TRABAJO EN EQUIPO & 6,05 & 6,75 & 7,40 & 8,25 & 8,21 & 8,63 \\
\hline & MOA04 & MEJORA CONTINUA & 5,45 & 5,91 & 6,39 & 5,24 & 6,46 & 6,73 \\
\hline & MOA06 & BÚSQUEDA OBJETIVOS EMPRESARIALES & 3,55 & 3,97 & 4,54 & 4,17 & 4,45 & 5,16 \\
\hline \multirow{3}{*}{ AMS_TEC } & MTF105 & NORMALIZACIÓN INFORMACIÓN & 6,05 & 6,08 & 6,51 & 7,01 & 7,29 & 7,27 \\
\hline & MTF107 & PROXIMIDAD DE LA INFORMACIÓN & 4,63 & 5,00 & 5,77 & 6,50 & 7,23 & 7,50 \\
\hline & MTF111 & MANEJO ADECUADO INFORMACIÓN DISPERSA & 4,26 & 4,68 & 5,34 & 6,45 & 6,55 & 7,08 \\
\hline
\end{tabular}

Por tanto, podemos afirmar que el modelo de SIIO propuesto para el sector de la edificación domótica se caracteriza por:

- Ser un sistema que mejora la descentralización de los agentes. Por tanto, se perciben como un sistema que mejora flexibilidad a la hora de trabajar en diferentes delegaciones o sedes de la empresa, así como el acceso a nuevos mercados salvando barreras geográficas. Esto debemos de entenderlo como búsqueda de nuevos mercados y relación comercial ya que, según hemos visto, las empresas consideran necesaria la proximidad geográfica a la ubicación del proyecto para trabajar en colaboración.

- Favorecer la identificación y evaluación de las tecnologías nuevas y emergentes que permitan lograr ventajas competitivas.

- Favorecer las relaciones cooperativas de ámbito nacional, en mayor medida, y de ámbito global en menor medida.

- Promocionar el trabajo en equipo y la aceptación de responsabilidades compartidas.

- Favorecer la mejora continua de los métodos y procesos.

- Favorecer la normalización de la información, minimizando la que no este en soporte electrónico y maneja adecuadamente la información generada en ubicaciones geográficamente dispersas. Este aspecto aunque es una característica percibida del SIIO propuesto, no es considerado, como hemos visto en la evaluación del modelo estructural, como un aspecto para la mejora de la flexibilidad y eficiencia organizativa.

Por otro lado, el SIIO propuesto presenta deficiencias en estos aspectos a mejorar:

- No se percibe que el plazo de entrega o puesta en el mercado, ni la rapidez para incorporar nuevos productos o servicios mejore gracias al SIIO. 
- No se percibe que sea fácil organizarse y cambiar la forma de trabajar.

- No se percibe que el SIIO reduzca coste y riesgos.

- No se considera que el sistema favorezca la toma de decisiones de forma descentralizada, y se sigue considerando una estructura centralizada.

- No se considera que el sistema potencie el aprendizaje colectivo.

- No se considera que el sistema favorezca la búsqueda permanente de un objetivo empresarial común en cada función o departamento.

\section{Limitaciones del trabajo de investigación}

El SIIO propuesto para el sector de la edificación trata de hacer frente a las necesidades de investigación planteadas. No obstante, existen limitaciones a considerar debidas a que la penetración de los sistemas de información en este tipo de empresas es bastante deficiente y no existe un concepto maduro formado sobre el SIIO. Por tanto, estos aspectos puede sesgar los resultados obtenidos hacia un perfil de empresa poco maduro desde el punto de vista de adopción de TIC y por tanto limita la generalización del estudio al tipo concreto de población identificada según el dimensionamiento del trabajo, para la cual sí se puede garantizar la sostenibilidad dado el amplio horizonte temporal de la investigación.

En el futuro serán necesarios estudios más concretos sobre el funcionamiento de los SIIO en el sector para generalizar sus implicaciones estratégicas (Carbonell Ureña, 2012); tales como el posible aumento del poder negociador de los clientes, derivado de la consolidación de consorcios de los principales compradores del sector, o el establecimiento o no de barreras de entrada, derivadas de la creación de redes interorganizacionales en el sector.

\section{Conclusiones}

El entorno actual de competitividad lleva a las empresas a buscar nuevas formas de competir. Los SIIO representan una alternativa en este sentido, puesto que permite incrementar los límites tradicionales de la organización traspasando las barreras naturales entre diferentes empresas y creando un vínculo que permita pasar de un estado de ventaja competitiva a una ventaja cooperativa.

Este estudio presenta tanto una componente documental como una fuerte componente de investigación por estar derivado de los resultados de las encuestas que se realizaron a estos agentes. Ello nos ha permitido identificar modelos de negocio así como las fuentes de creación de valor. Un SIIO permite redefinir a las organizaciones bajo condiciones de flexibilidad y eficiencia que son dos pilares que le permitirán competir en el entorno actual. Con este trabajo hemos pretendido reflejar la importancia que tiene para las empresas definir su negocio a través de un modelo que garantice la viabilidad presente y futura de la empresa. 


\section{Referencias bibliográficas}

BIC-GALICIA. (2010). Guía sectorial de la construcción.

Carbonell-Ureña, J. F. (2012). "Propuesta de un modelo de integración para la gestión de la cadena de suministro en el sector de la construcción". Tesis Doctoral. Universidad Politécnica de Valencia.

Castro-Lacouture, D., Medaglia, A. L., \& Skibniewski, M. (2007). Supply chain optimization tool for purchasing decisions in B2B construction marketplaces. Automation in Construction. Elsevier Science Publishers, 16, 569-575.

Castro-González, I. (2008). "Comparativa de tecnologías domóticas en la elaboración de proyectos para la gestión integrada de servicios del hogar digital". Proyecto Fin de Carrera dirigido por Antonio Pereira-Rama. Universidad de Vigo.

Cepeda-Carrión, G., \& Roldán-Salgueiro, J. L. (2005). Aplicando en la práctica la técnica PLS en la Administración de Empresas. Paper presented at the XV Congreso Anual de la Asociación Científica de Economía y Dirección de la Empresa (ACEDE).

Cinza-Cabarcos, B. E. (2008). "Propuesta práctica de instalación domótica basada en tecnología X-10 orientada a la mejora del ahorro energético en el hogar como medida para la optimización del consumo global de energía en el ámbito doméstico". Proyecto Fin de Carrera dirigido por Antonio Pereira-Rama. Universidad de Vigo.

Criado-Fernández, M. (2000). "Caracterización de modelos de cooperación entre organizaciones como base para la obtención de estructuras flexibles y competitivas". Tesis Doctoral. Universidad Politécnica de Madrid.

Chung, J. K. H., Kumaraswamy, M. M., \& Palaneeswaran, E. (2009). Improving megaproject briefing through enhanced collaboration with ICT. Automation in Construction. Elsevier Science Publishers, 18(7), 966-974.

Jeong, Y.-S., Eastman, C. M., Sacks, R., \& Kaner, I. (2009). Benchmark tests for BIM data exchanges of precast concrete. Automation in Construction. Elsevier Science Publishers, 18(4), 469-484.

Kerridge, S., Halaris, C., \& Mentzas, G. (2000). "SupplyPoint: An Integrated System for Supporting e-Business in the Construction Sector". University of Sunderland, United Kingdom.

Kong, S. C. W., Li, H., Hung, T. P. L., Shi, J. W. Z., Castro-Lacouture, D., \& Skibniewski, M. (2004). Enabling information sharing between E-commerce systems for construction material procurement. Automation in Construction. Elsevier Science Publishers, 13, 261-276.

Lockett, N. J., \& Brown, D. H. (2006). Aggregation and the Role of Trusted Third Parties in SME E-Business Engagement: A Regional Policy Issue. International Small Business Journal, 24(4), 379-404. 
Lozano-Santiago, S. (2007). Validación de un modelo de medida de las dificultades en los procesos de toma de decisiones sobre la carrera profesional. Revista de Educación, 343, pp. 325-351.

Orero-Giménez, A., \& Criado-Fernández, M. (1999). El Sistema de Información Interorganizacional como fuente y soporte de ventajas competitivas. Paper presentado en el IX Congreso Nacional de la Asociación Científica de Economía y Dirección de Empresa (ACEDE).

Pereira-Rama, A., \& Chaparro-Peláez, J. (2005). La gestión integrada de servicios del Hogar Digital soportada por un sistema de información interorganizacional. Paper presentado en el XIX Congreso Hispano Francés de la Asociación Europea de Dirección y Economía de la Empresa (AEDEM).

Pizarro-Moreno, I., Real, J. C., \& De la Rosa, M. D. (2011). La incidencia del capital humano y la cultura emprendedora en la innovación. Cuadernos de Economía y Dirección de la Empresa, 14, 139-150.

Schlueter, A., \& Thesseling, F. (2009). Building information model based energy/exergy performance assessment in early design stages. Automation in Construction. Elsevier Science Publishers, 18(2), 153-163.

Shin, Y., An, S.-H., Cho, H.-H., Kim, G.-H., \& Kang, K.-I. (2008). Application of information technology for mass customization in the housing construction industry in Korea. Automation in Construction. Elsevier Science Publishers, 17(7), $831-838$.

Telefónica. (2004). "Libro Blanco del Hogar Digital y las Infraestructuras Comunes de Telecomunicación". Fundación Telefónica.

Vanlande, R., Nicolle, C., \& Cruz, C. (2008). IFC and building lifecycle management. Automation in Construction. Elsevier Science Publishers, 18(1).

Yeung, J. F. Y., Chan, A. P. C., \& Chan, D. W. M. (2009). A computerized model for measuring and benchmarking the partnering performance of construction projects. Automation in Construction. Elsevier Science Publishers, 18(8).

White, A., Daniel, E., Ward, J., \& Wilson, H. (2007). The adoption of consortium B2B e-marketplaces: An exploratory study. Journal of Strategic Information Systems, $16,71-103$. 